made to our diagnostic/treatment pathway between these 2 years.

Results Between 2011 and 2012, number of patient diagnosed with NSCLC increased from 140 to 162 . Patients presenting with stage 1-3a and good PS 0-2 increased from 33(23.5\%) to 43(26.5\%), largely due to increased stage 1 disease (table 1$)$. In these patients, cough was a predominant symptom in $44 \%$ (2011) and 68\% (2012). In 2011, 8(24\%) patients presented to GP with unexplained cough as only symptom, with average symptom length 7.5 months. In 2012, 18(42\%) presented with unexplained cough, 14 through GP with average symptom length 3.3 months, in 7 cases under 1 month.

$\begin{aligned} & \text { Abstract P5. Table 1. Comparison of patients diagnosed with } \\
& \text { lung cancer through our thoracic oncology service in }\end{aligned}$
\begin{tabular}{lll}
$\mathbf{2 0 1 2}$ & $\mathbf{2 0 1 1}$ and \\
\hline Year & $\mathbf{2 0 1 1}$ & $\mathbf{2 0 1 2}$ \\
\hline No. diagnosed with lung cancer & 173 & 200 \\
No. diagnosed non-small cell lung cancer (NSCLC) & 140 & 162 \\
No. NSCLC with Stage 1-3a (\%) & $38(27 \%)$ & $51(31.5 \%)$ \\
No. Stage 1a\&b & 12 & 21 \\
No. Stage 2a\&b & 10 & 12 \\
No. Stage 3a & 16 & 18 \\
No. NSCLC Stage 1-3a \&good ECOG & $33(23.5 \%)$ & $43(26.5 \%)$ \\
performance status (PS) 0-2 & & \\
No. NSCLC Stage 1-2b \& PS 0-2 & $20(14 \%)$ & $31(19 \%)$ \\
\% NSCLC radically treated & $27(19 \%)$ & $40(25 \%)$ \\
No. Surgical resection & 16 & 26 \\
No. Radicalchemoradiotherapy/ radiotherapy & 11 & 14 \\
\hline
\end{tabular}

Conclusions Our results mirror national increase in lung cancer diagnosed. We have seen an increased proportion of early stage disease, especially stage 1 . Radical treatment rates increased by $6 \%$, with curative surgery the main modality. Cough was a predominant symptom in patients presenting with radically-treatable NSCLC to their GP, and was the major focus of the national public awareness campaign in 2012. Locally, not only has the number of patients presenting with unexplained cough as a primary symptom increased between 2011 and 2012, but they are presenting sooner. It is possible therefore that the national campaign has contributed to detection of earlier stage disease in 2012 and translation to higher radical treatment rates. It will be interesting to see whether these initiatives have had similar effects on a national level and we await the LUCADA results for 2012.

\section{P6 TEMPORAL RELATIONSHIPS BETWEEN LUNG CANCER MDT RECOMMENDATIONS AND FINAL OUTCOMES}

${ }^{1} \mathrm{~K}$ Shah, ${ }^{1} \mathrm{~L}$ Fairclough, ${ }^{2} \mathrm{R}$ Sundar, ${ }^{3} \mathrm{SCO}$ Taggart; ${ }^{1}$ University of Manchester, Manchester, UK; ${ }^{2}$ Wrightington, Wigan \& Leigh NHS Foundation Trust, Wigan, UK; ${ }^{3}$ Salford Royal NHS Foundation Trust, Salford, UK

\subsection{6/thoraxjnl-2013-204457.156}

Introduction and Objective A previous study [1] has identified a discordancy rate of $33 \%$ between MDT treatment decision and actual treatment given to patients not suitable for best supportive care. The Northwest Sector Lung Cancer MDT (SMDT) provides recommendations for treatment for approximately 700 new diagnoses/year attending the Salford, Wigan and Bolton catchment area. The Salford Service is centralised around the
Lead Clinician who assesses and refers $>90 \%$ of patients to SMDT (c.f. $<50 \%$ Wigan \& Bolton). A detailed proforma is completed for each patient detailing performance score, co-morbidity and all diagnostic data. This study sets out to determine the temporal rates of discordance between SMDT recommendations and actual treatment pursued for Salford and Wigan.

Method Using a 1:2 random selection of all patients from Salford \& Wigan diagnosed in 2011 and 2012, the hospital notes were examined to identify the SMDT recommendation and subsequent treatment received by the patient. Patients referred for Best Supportive Care were excluded. Any discordance was further explored by review of the notes.

Results The study identified a total of 441 patients; 213 (Salford) and 228 (Wigan). The discordance rates for Salford were 8\% (2011), $12.8 \%$ (2012) \& 10.4\% (combined) c.f. 26.9\% (2011), $36.9 \%$ (2012) and $32.4 \%$ for Wigan. The overall SMDT discordancy rate was $17.7 \%$ (2011), $27.0 \%$ (2012) \& 19.9\% (combined). Discordance occurred most commonly due to co-morbidity health concerns (44.6\%), patient wishes (15.7\%) and as a result of new clinical information becoming available (27.7\%).

Conclusions

1. SMDT recommendations are dependent on a detailed knowledge of patient wishes, co-morbidity and stage of disease.

2. Discordance reflects weaknesses in patient assessment prior to referral to SMDT.

3. Centralisation of assessment before referral to SMDT is associated with improved and acceptable discordancy rates.

4. We recommend assessment by a dedicated Lung Cancer Clinician ahead of referral to SMDT.

\section{REFERENCE}

1. Pemberton $L$ et al. Do treatment decisions made at lung cancer multi-disciplinary team meetings (MDTs) reflect the actual treatment given in practice? Lung Cancer, Volume 79, Supplement 1, January 2013, Page $\$ 36$.

\section{P7 RADICAL TREATMENT FOR LUNG CANCER: IMPACT OF RECENT SERVICE IMPROVEMENTS}

${ }^{1} \mathrm{M}$ Gornasa, ${ }^{2} \mathrm{H}$ Essa, ${ }^{3} \mathrm{H}$ Sheikh, ${ }^{4} \mathrm{R}$ Shah, ${ }^{1} \mathrm{SCO}$ Taggart; ${ }^{1}$ Salford Royal NHS Foundation Trust, Manchester, UK; ${ }^{2}$ University of Manchester, Manchester, UK; ${ }^{3}$ Christie Hospital, Manchester, UK; ${ }^{4}$ University Hospital South Manchester, Manchester, UK

\subsection{6/thoraxjnl-2013-204457.157}

Introduction The Salford Lung Cancer Service (SLCS) has introduced a number of improvements in care including PET-CT scanning (2005), EBUS (2008), a Northwest Sector MDT for treatment decisions (2009), CPEX testing (2009), IMRT/stereotactic radiotherapy (2011) and development of a surgical high risk MDT (2011).

The SLCS holds a data-base of all patients having surgery or radical radiotherapy (DXT) each year since 2000. This study sets out to review our performance for patients receiving either surgery or radical DXT from 2005 to 2012.

Methods The data-base was used in parallel to reviewing each patient record to determine surgical procedure, presence or absence of pre-operative or pre-DXT histology and final tumour stage. For comparative analysis of the effect of these new developments on service delivery, each year was pooled into 2 main groups; Group A (2005-2008) and Group B (2009-2012). Tests for significance were performed using Chi-Squared tables at 5\% level.

Results Overall, a total of 178 patients underwent surgery and 138 DXT (See Table). Patients receiving surgery or DXT 